\title{
Inquérito sobre a prevalência de bócio endêmico no Brasil em escolares de 6 a 14 anos: 1994 a $1996^{1}$
}

\author{
Heleno Rodrigues Corrêa Filho, ${ }^{2}$ João Batista Furtado Vieira, ${ }^{3}$ \\ Yara Simoni Pereira Silva, ${ }^{3}$ Giovanini Evelim Coelho, ${ }^{3}$ Felicidade \\ dos Anjos Carvalho Cavalcante ${ }^{3}$ e Maria da Paz Luna Pereira ${ }^{3}$
}

RESUMO Objetivo. Determinar as prevalências estaduais e nacional do bócio endêmico relacionado com a deficiência de iodo em escolares de 6 a 14 anos, com base em dados obtidos no inquérito nacional realizado de 1994 a 1996 no Brasil.

Materiais. Em 428 municípios de todos os estados brasileiros, o exame de inspeção-palpação foi utilizado para coletar dados de 178774 escolares de 6 a 14 anos. Uma sub-amostra foi selecionada para determinação do conteúdo de iodo na urina e sal culinário residencial.

Resultados. Em comparação com dados de 1975, último inquérito antes deste aqui relatado, constatou-se queda de 86\% na prevalência mediana de bócio graus I e II (de 12 para 1\%). Em $21 \%$ dos municípios, a prevalência observada foi zero. Das 16803 amostras urinárias para dosagem de iodo foi possível recuperar 7702 e associá-las às respectivas fichas clínicas individuais (perda de 54\%). A mediana de iodo urinário foi de 14,0 $\mu \mathrm{g} / \mathrm{dL}$ e não se verificou correlação significativa com os dados clínicos de bócio. Os estados do Acre, Amapá e Tocantins tiveram mediana populacional de excreção urinária de iodo igual ou abaixo de 9,0 $\mu \mathrm{g} / \mathrm{dL}$. Em três municípios a dosagem urinária de iodo foi gravemente baixa $(<2,5 \mu \mathrm{g} / \mathrm{dL})$ em todas as amostras: Paranã (Tocantins); Conceição (Paraíba); e Nova Roma (Goiás). O sal consumido nas residências (458 amostras coletadas) apresentou sub-dosagem de iodo suplementar, com $50 \%$ abaixo de $20 \mathrm{mg} / \mathrm{kg}$ ou $20 \mathrm{ppm}$. Em 7\% das amostras, a dosagens de iodo foi menor do que $10 \mathrm{mg} / \mathrm{kg}$ de sal, inclusive em estados produtores de sal, como o Rio Grande do Norte.

Conclusões. Há evidências de expansão da área endêmica de bócio para as regiões de abertura de fronteira agrícola, com exposição de crianças a dieta doméstica carente de iodo veiculado pelo sal. Esta carência associou-se com o aumento de risco para bócio $(O R=1,85 ; 1,68<O R<2,03$; IC 95\% Cornfield) nas áreas onde a iodação do sal consumido foi deficiente (<10 mg/kg).

Palavras-chave Bócio endêmico, epidemiologia; inquéritos de morbidade; inquéritos nutricionais; iodo, provisão \& distribuição; estudos transversais; estudos epidemiológicos.

Em 1994, após quase 20 anos da realização do último inquérito de abran- gência nacional sobre prevalência do bócio, o Ministério da Saúde do Brasil

viados a este autor no seguinte endereço: UNICAMP, DMPS, Cidade Universitária Zeferino Vaz, CEP 13084-970, Campinas, SP, Brasil. E-mail: helenocf@unicamp.br

Contrato UNICEF - BRZ 591/PER/TEMP/ 0201. dade de Ciências Médicas, Departamento de Medicina Preventiva e Social, Laboratório de Aplicação de Epidemiologia, Campinas, SP. Correspondência e pedidos de separatas devem ser en-
Ministério da Saúde, Fundação Nacional de Saúde, Brasília, Brasil. decidiu realizar novo inquérito (1). Conforme os dados obtidos em 1975, a prevalência média de bócio no território nacional era de $14 \%$ entre escolares de 7 a 14 anos, tendo o país, na época, 25 estados, incluindo o Distrito Federal. A prevalência mínima observada naquela ocasião foi de $0,7 \%$, no Rio 
Grande do Norte; a máxima, 33\%, na Bahia. As áreas endêmicas detectadas apontavam para o maciço cristalino brasileiro da região Centro-Oeste e zonas rurais afastadas da Bahia, Minas Gerais, Mato Grosso (que cobria também o atual Estado de Mato Grosso do Sul, que só foi criado em 1977) e Pará, além da divisão serrana entre os estados do Piauí e Maranhão. A mediana para o país era de $12 \%$. A prevalência registrada anteriormente, em 1955, havia sido de $21 \%$, sendo que o inquérito de 1975 detectou uma queda de $32 \%$ nos níveis de prevalência dentro daquele período de 20 anos.

No Brasil, o solo é caracterizado por relevos de áreas geológicas antigas, erodidas e de baixo teor de iodo solúvel nas camadas superficiais. A queda ocorrida de 1955 até 1975 foi atribuída à implementação de políticas de suplementação de iodo através do sal de cozinha, preconizando-se legislação que tornou obrigatória a adição de iodato de potássio a todo o sal produzido para consumo humano em território brasileiro. A expectativa de queda continuada nos níveis de prevalência do bócio, expressando o controle das doenças por deficiência de iodo, motivou a realização de novo inquérito, visto que a doença continuava a ser diagnosticada no país, embora em níveis menores que anteriormente. Além disso, buscavam-se dados que apoiassem ou retificassem as políticas oficiais de suplementação. Sendo o sal de cozinha o veículo escolhido para administrar o suplemento de iodato de potássio, a dosagem de iodo no sal foi analisada no inquérito de 1994 para avaliação de sua eficácia, limites e deficiências prováveis.

Para a realização do inquérito nacional de prevalência de 1994, uniram forças e recursos a Fundação Nacional de Saúde (FNS), o Instituto Nacional de Alimentação e Nutrição (INAN) e o Fundo das Nações Unidas para a Infância (UNICEF). Assim, o objetivo do presente trabalho foi determinar as prevalências estaduais e nacional do bócio endêmico relacionado com a deficiência de iodo com base nos dados clínicos de exames de inspeção e palpação em escolares de 6 a 14 anos, ob-

TABELA 1. Distribuição dos municípios selecionados por estado segundo a característica intencional ou probabilística da escolha. Inquérito nacional sobre prevalência de bócio, Brasil, 1994 a 1996

\begin{tabular}{|c|c|c|c|c|}
\hline Estado & $\begin{array}{c}\text { Município- } \\
\text { sentinela }\end{array}$ & Vizinhos $^{a}$ & Sorteio & Total \\
\hline Pará & 3 & 9 & 13 & 25 \\
\hline Tocantins & 4 & 28 & 15 & 47 \\
\hline Maranhão & 3 & 8 & 14 & 25 \\
\hline Bahia & 1 & 5 & 13 & 19 \\
\hline Goiás & 4 & 19 & 13 & 36 \\
\hline Minas Gerais & 2 & 6 & 14 & 22 \\
\hline Cada um dos outros 20 estados $^{b}$ & - & - & 20 & 284 \\
\hline Total & 17 & 75 & - & 458 \\
\hline
\end{tabular}

a Municípios vizinhos ao município-sentinela.

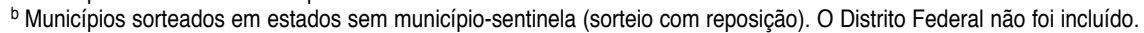

tidos a partir do inquérito nacional realizado de 1994 a 1996 no Brasil. Pretende-se ainda fazer uma comparação histórica para 17 municípiossentinela selecionados como tal pela vigilância epidemiológica nacional.

\section{MATERIAIS E MÉTODOS}

Optou-se por avaliar o bócio utilizando a classificação clínica de inspeção e palpação da Organização Mundial da Saúde (OMS) em três níveis (2), complementada pela dosagem do teor de iodo urinário e iodo no sal de cozinha domiciliar em sub-amostra de escolares, embora a classificação de Gandra (3) viesse sendo utilizada nos municípios-sentinela. Por razões de exeqüibilidade, foram descartadas a ultrasonografia volumétrica da tireóide e a dosagem de hormônio estimulador da tireóide (TSH) neonatal, dentre outros métodos com maior acurácia e reprodutibilidade.

A equipe que coordenou a execução do inquérito optou por analisar a cobertura da faixa etária de 6 a 14 anos utilizando esquema classificatório de três níveis (0-I-II) com exame de inspeção clínica visual e palpação. ${ }^{4}$ Fo-

\footnotetext{
4 Brasil, Ministério da Saúde, Instituto Nacional de Alimentação e Nutrição, Fundação Nacional de Saúde. Programa Nacional de Combate ao Bócio Endêmico: plano de ação para 1994 [documento interno mimeografado]. Brasília, DF: Instituto Nacional de Alimentação e Nutrição/Fundação Nacional de Saúde; 1994. Pp. 1-13.
}

ram discutidas e excluídas outras alternativas a partir de um estudo piloto que considerou outras faixas ( 8 a 10 e 6 a 12 anos). A escolha vai ao encontro das recomendações de Gandra (3) para a faixa etária de 9 a 14 anos e de Vitti et al. (4) para a faixa de 6 a 14 anos como grupo vulnerável indicador para o bócio endêmico.

Para os estados onde existiam municípios-sentinela, foram incluídos os 17 municípios-sentinela ${ }^{5}$ do Programa Nacional de Controle de Doenças Carenciais, além dos municípios limítrofes de cada município-sentinela - resultando, na época, em outros 75 municípios (tabela 1). A este grupo foi acrescentada amostra aleatória, através de método de seleção proporcional ao tamanho da população. Deste modo, a amostra foi caracterizada como predominantemente intencional, visando cobrir as áreas onde se supunha maior a prevalência de bócio.

Para cada estado onde não existiam municípios-sentinela, estabeleceu-se como meta sortear 20 municípios por método aleatório com reposição. Esta repetição propiciou números menores do que 20 municípios por estado. Estados com municípios populosos tiveram as maiores cidades repetidas

\footnotetext{
5 Brasil, Ministério da Saúde, Fundação Nacional de Saúde, Gerência de Endemias Focais. Dados sobre prevalências de bócio endêmico em 1984, 1986, 1990 nos municípios sob vigilância (municípiossentinela) [documento mimeografado]. Brasília: Fundação Nacional de Saúde; 1994. Pp. 1-15.
} 
mais freqüentemente, por tratar-se de amostra proporcional ao tamanho populacional. Assim sendo, ao invés de 400, a amostra incluiu 284 municípios sorteados em estados onde não havia municípios-sentinela. Estes foram somados aos 174 situados nos demais estados, totalizando a amostra prevista de 458 municípios (tabela 1).

Inicialmente, planejou-se estudar, em cada município, cinco escolas sorteadas aleatoriamente, sendo duas em área urbana e três em área rural. Em municípios com menos de cinco escolas todas as escolas seriam estudadas. Contudo, o número de escolas investigadas não foi o previsto por município, havendo casos em que alunos de todas as escolas de grandes municípios foram examinados, o que não prejudicou o plano amostral ou a análise. Foram amostradas crianças de 1823 escolas brasileiras.

Em cada município foram sorteadas 40 crianças para dosagem de iodo urinário. Dentre elas, as 20 primeiras foram visitadas para coleta de amostras de sal residencial. Também foram coletadas amostras da fonte comercial utilizada como suprimento pela família da criança.

\section{Execução e controle da coleta de dados}

Foram convocados e treinados 59 médicos da FNS para: a) aspectos clínicos da anatomia e fisiologia da tireóide e técnicas semióticas de inspeção e palpação para diagnóstico de bócio; b) aspectos práticos, em grupos de até cinco médicos, em área endêmica do Estado de Goiás. Os médicos foram padronizados por monitores selecionados por critério de experiência de 10 anos em inquéritos populacionais.

A maioria dos estados finalizou o levantamento até dezembro de 1995. Bahia, Rio Grande do Sul e Mato Grosso somente conseguiram concluir o inquérito em 1996.

As planilhas de cada estado foram encaminhadas para a assessoria técnica gerencial do gabinete do Ministro da Saúde em Brasília (DF), para consolidação geral. As fichas individuais foram então digitadas através da gerência técnica de endemias focais, em 1996, em módulo de base de dados computacional preparado pela empresa de processamento de dados do Sistema Único de Saúde (DATASUS). Nesta digitação foram incluídas as dosagens de iodo no sal de cozinha.

A base de dados com fichas individualizadas disponíveis para processamento ficou pronta em agosto de 1997, contendo 178774 registros, aos quais foram acoplados registros individuais de iodo urinário. Foram posteriormente associados os dados de 5833 crianças que tiveram o iodo dosado no sal doméstico e, em alguns casos, também na fonte comercial de sal apontada pela família. A análise de iodo no sal de consumo residencial foi feita como referência ecológica por município (aplicando a cada criança o valor mediano de todas as dosagens amostradas no município, e não a dose verificada na sua própria residência), tendo em vista as dificuldades para a identificação individual das amostras.

\section{Processamento e análise dos dados}

Os coeficientes de prevalência por estado foram calculados através da média ponderada das prevalências municipais encontradas nos municípios pesquisados. A descrição nacional resulta destas prevalências. Cada prevalência municipal foi multiplicada pela proporção que a população de 6 a 14 anos representava em relação à mesma faixa etária do estado considerando as proporções médias de 3 anos - 1994 a 1996. Os coeficientes municipais foram calculados de modo direto. Buscou-se correlacionar os dados clínicos individuais com as dosagens de iodo urinário e com a dose mediana de iodo no sal do município de residência. Os dados assim obtidos foram comparados quanto às suas freqüências e correlações totais e parciais segundo a idade.

Foram consideradas como indicadores de bócio as classificações em grau I e II segundo o Manual de Combate aos Distúrbios por Deficiência de Iodo no Brasil (5) e a OMS (6). O grau endê- mico leve (ou médio) (5) de bócio foi considerado para prevalências de 5,0 a 19,9\%; o grau endêmico moderado foi considerado para prevalências de 20,0 a $29,9 \%$; e o grau endêmico grave foi considerado para prevalências acima de $30,0 \%$ (7).

As doses de iodo urinário foram classificadas em normais ou suficientes ( $\leq 10 \mu \mathrm{g} / \mathrm{dL}$ ), deficiência leve (5-10 $\mu \mathrm{g} / \mathrm{dL})$, moderada $(2,5-5 \mu \mathrm{g} / \mathrm{dL})$ e grave $(<2,5 \mu \mathrm{g} / \mathrm{dL})$ (7). As dosagens foram efetuadas segundo metodologia descrita por Dunn et al. (8).

\section{RESULTADOS}

Foram obtidos dados de 428 municípios do território brasileiro, tendo sido examinados 178774 escolares de 6 a 14 anos. O total de perdas foi de 30 municípios dentre os 458 inicialmente previstos $(7 \%)$.

A prevalência média nacional de bócio em escolares de 6 a 14 anos nos 428 municípios amostrados, agregados os escolares de zonas urbana e rural, foi de $4 \%$, com variância de 40,98 e erro padrão da amostra de 0,309. A distribuição desviada, ou "concentrada" à esquerda, torna preferencial a análise não paramétrica para resumir a situação dos estados onde o inquérito foi realizado.

A mediana nacional foi de 1,3\%, com distribuição dos valores de quartis em Q1 = 0,3; Q3 = 4,6; mínimo = 0,0; máximo $=47,2$, considerando-se os 26 estados em sua distribuição do tipo "diagrama de cinco valores" (9). Dos 26 estados brasileiros pesquisados, $50 \%$ apresentaram prevalências medianas entre 0,3 e 4,6\%. Tanto o critério não paramétrico (medianas) quanto o paramétrico (médias) apontaram os estados de Rondônia e Mato Grosso do Sul como os que apresentaram valores aberrantes em relação ao país. As medianas estaduais de prevalência total de bócio grau I e II (apenas 94 crianças com grau II em todo o inquérito) aparecem na tabela 2. Os números apresentados estão ponderados pela importância populacional dos municípios dentro dos estados. Nesta análise, os estados com mediana igual ou 
TABELA 2. Prevalência total de bócio entre escolares de 6 a 14 anos. Inquérito nacional, Brasil, 1994

\begin{tabular}{|c|c|c|c|}
\hline Estado & Número de municípios & Mediana & Crianças examinadas \\
\hline Acre & 5 & 9,4 & 818 \\
\hline Amazonas & 11 & 11,5 & 7386 \\
\hline Roraima & 7 & 0,0 & 10069 \\
\hline Amapá & 8 & 1,3 & 5018 \\
\hline Pará & 23 & 5,0 & 7874 \\
\hline Rondônia & 6 & 20,0 & 5395 \\
\hline Tocantins & 41 & 0,5 & 8175 \\
\hline Maranhão & 25 & 3,2 & 7554 \\
\hline Piauí & 16 & 3,4 & 4823 \\
\hline Ceará & 15 & 3,6 & 5370 \\
\hline Rio Grande do Norte & 17 & 1,8 & 9358 \\
\hline Paraíba & 18 & 1,6 & 3814 \\
\hline Pernambuco & 18 & 1,0 & 8580 \\
\hline Alagoas & 15 & 0,0 & 5656 \\
\hline Sergipe & 16 & 0,2 & 7084 \\
\hline Bahia & 18 & 0,2 & 4666 \\
\hline Goiás & 36 & 2,8 & 9841 \\
\hline Mato Grosso do Sul & 15 & 20,1 & 6708 \\
\hline Mato Grosso & 17 & 10,2 & 6415 \\
\hline Paraná & 17 & 6,1 & 10461 \\
\hline Santa Catarina & 16 & 0,1 & 4356 \\
\hline Rio Grande do Sul & 10 & 1,0 & 4809 \\
\hline São Paulo & 15 & 1,6 & 11673 \\
\hline Espírito Santo & 13 & 5,0 & 3282 \\
\hline Rio Janeiro & 11 & 1,0 & 9242 \\
\hline Minas Gerais & 19 & 1,1 & 10347 \\
\hline Total & 428 & 1,3 & 178774 \\
\hline
\end{tabular}

acima de $20 \%$ são Mato Grosso do Sul e Rondônia.

\section{Situação dos municípios-sentinela}

A FNS mantinha 17 municípios-sentinela onde tradicionalmente era feito inquérito de prevalência (10), sendo os últimos dados comparativos disponíveis de 1990 (11). Estes dados podem ser cotejados com os dados de prevalência total obtidos no inquérito nacional de 1994 (tabela 3). Verifica-se que o único estado onde não ocorreu redução de prevalência em municípiosentinela foi o do Maranhão. A situação geral dos municípios brasileiros é apresentada na tabela 4.

Os municípios estudados com prevalência grave $(4,4 \%)$ e moderada $(0,7 \%)$, não se distribuem de modo homogêneo no território brasileiro (tabela 5). Oitenta e três municípios apresentaram prevalências entre 5 e $20 \%$, classificadas como endemia leve (ta- bela 6). Não foram detectadas prevalências endêmicas graves de bócio em escolares das regiões da fronteira norte do país - Roraima, Amazonas e Amapá. A situação geográfica global do país aparece na figura 1.

\section{Dosagens de iodo urinário}

Os métodos empregados na titulação do iodo urinário foram descritos em documentos disponíveis no INAN, junto à coordenação do programa de controle do bócio endêmico. ${ }^{6}$ Foram tomadas 16803 dosagens distribuídas nos 428 municípios da amostra. Destas, foi possível recuperar 9817 e identificar e digitar um total de 7702 registros, representando uma recuperação

\footnotetext{
6 Maciel RMB. Inquérito para avaliação da prevalência de bócio em escolares brasileiros: métodos para dosagem de iodo urinário em inquéritos populacionais [documento mimeografado]. São Paulo: Universidade Federal do Estado de São Paulo/ Escola Paulista de Medicina; 1994.
}

de $46 \%$. As perdas de identificação ao digitar foram atribuídas a dificuldades na identificação de crianças e municípios. Não foi possível recuperar 9101 fichas de dosagens de iodo urinário, uma vez que existe menção da coleta e dosagem de 16803 amostras (12).

Os valores das dosagens de iodo urinário em crianças comparados com os dados clínicos individuais não apresentaram correlação significativa (coeficiente de correlação parcial de Pearson $=-0,00124 ; P=0,91 ; \mathrm{n}=7$ 682).

A representação do território nacional foi díspar, não idêntica à dos municípios que constam da lista coletada de exames de inspeção e palpação. Isto ocasiona desproporcionalidade relativa tanto à amostra do inquérito clínico quanto ao peso populacional a ser multiplicado para ponderar as prevalências regionais. Um exemplo desta desproporcionalidade é que o Estado do Acre está representado por apenas um município (Brasiléia).

Dentro do grupo de dosagens obtidas, procurou-se saber a distribuição das dosagens de iodo segundo a classificação clínica de bócio das crianças examinadas (tabela 7).

Não foram observados, em nível individual, indícios de relação entre os graus clínicos de bócio e a excreção de iodo urinário. É importante salientar que, em crianças, esta correlação não seria esperada durante a prépuberdade, uma vez que o desenvolvimento de sintomas clínicos é conseqüente ao período carencial, que, neste caso, não pôde ser especificado como tendo iniciado na infância precoce, dadas as condições de iodação de sal no Brasil até o ano do inquérito. Devese considerar que os níveis de excreção leve, moderada e grave são expressivos $(33 \%)$ e que a carência de iodo leva tempo para se expressar em crianças em fase de crescimento em termos de crescimento glandular palpável ou visível.

A média global nacional de excreção urinária de iodo obtida nas amostras foi de 15,34 $\mu \mathrm{g} / \mathrm{dL}$, com variância de 97,558 e desvio padrão de 9,877 ( $\mathrm{n}=$ 7 652). Esta distribuição, no entanto, não é normal, apresentando-se desviada para a esquerda, o que propicia 
TABELA 3. Prevalência de bócio total entre escolares de 6 a 14 anos em municípiossentinela de seis estados em 1990 e 1994

\begin{tabular}{lrr}
\hline \multirow{2}{*}{ Município/estado } & \multicolumn{2}{c}{ Prevalência (\%) } \\
\cline { 2 - 3 } \multicolumn{1}{c}{ Jequitinhonha/Minas Gerais } & 1990 & $1994^{\mathrm{a}}$ \\
\hline Mirabela/Minas Gerais & 39,40 & 10,00 \\
Palmeiras de Goiás & 29,50 & 0,44 \\
Flores de Goiás & 25,00 & 0,59 \\
Nova Roma/Goiás & 26,90 & 1,39 \\
Balsas/Maranhão & 17,40 & 2,29 \\
Riachão/Maranhão & - & 9,52 \\
São Raimundo das Mangabeiras/Maranhão & 16,46 & 19,56 \\
Cametá/Pará & 19,50 & 19,07 \\
Oeiras do Pará & 19,20 & 2,79 \\
Peixe/Tocantins & 20,75 & 8,52 \\
Natividade/Tocantins & 39,90 & 1,20 \\
Araguaina/Tocantins & 28,60 & 1,07 \\
Porto Nacional/Tocantins & - & 0,66 \\
Correntina/Bahia & 11,00 & 0,00 \\
& - & 0,00 \\
\hline
\end{tabular}

Fonte: Fundação Nacional de Saúde, 1994 (padrão GANDRA-0B).

a Dados não disponíveis para dois dos 17 municípios-sentinela (Cabeceiras, Estado de Goiás, e Limoeiro do Ajuru, Estado do Pará).

TABELA 4. Distribuição dos valores municipais de prevalência de bócio em escolares de 6 a 14 anos. Inquérito nacional, Brasil, 1994 a 1996

\begin{tabular}{lcr}
\hline Prevalência de bócio & Freqüência & \multicolumn{1}{c}{$\%$} \\
\hline$\leq 5,0$ (não endêmico) & 326 & 76,18 \\
5,0 a 19,9 (leve) & 83 & 19,39 \\
20,0 a 29,9 (moderado) & 15 & 3,50 \\
$\geq 30,0$ (grave) & 4 & 0,93 \\
Total & 428 & 100,00 \\
\hline
\end{tabular}

a construção de intervalo de confiança de $95 \%$ (IC 95\%) estendido de $+35,09$ $\mu \mathrm{g} / \mathrm{dL}$ até $-4,41 \mu \mathrm{g} / \mathrm{dL}$. O inconveniente de utilizar este estimador global do país para testes é a perturbação (nuisance) destes valores negativos, onde estaria incluída a faixa de gravidade para o referencial adotado no inquérito $(0<\mathrm{x} \mu \mathrm{g} / \mathrm{dL}<2,0 \mu \mathrm{g} / \mathrm{dL})$. Para evitar a amplitude desta distribuição, optou-se por trabalhar com a distribuição dos estimadores e avaliadores não paramétricos. A tabela 8 exibe as medianas de dosagens de iodo urinário. A distribuição dos valores medianos permite visualizar a distribuição dos estimadores de nível de iodo urinário e localizar quais, dentre os estados pesquisados, são aqueles que merecem atenção epidemiológica prioritária. A distribuição das dosagens segundo um diagrama de cinco valores (9) em unidades de $\mu \mathrm{g} / \mathrm{dL}$ apresenta mediana de 14,0 e distribuição em quartis de Q1 = 8,1; Q3 = 21,4 ; mínimo $=0,0$ e máximo $=97,0$. Estes valores mostram os possíveis limites para intervenção em programas preventivos e seus componentes de vigilância epidemiológica.

Se trabalharmos com valores medianos, utilizando o primeiro quartil (Q1), ou mesmo o intervalo de confiança paramétrico, o limiar de 8,1 justifica estabelecer que os estados abaixo de 10 $\mu \mathrm{g} / \mathrm{dL}$ de excreção sejam considerados de nível endêmico leve e, portanto, a eles seja dirigido o estabelecimento de municípios-sentinela, além da intensificação das medidas propostas em pro- grama nacional de controle. Desta maneira, os estados e municípios com medianas próximas de $8,1 \mu \mathrm{g} / \mathrm{dL}$ (conforme o primeiro quartil), ou, ainda, aqueles com deficiência endêmica leve na excreção de iodo $(<10 \mu \mathrm{g} / \mathrm{dL})(6,7)$ deveriam ser considerados como os mais problemáticos: Acre, Amapá e Tocantins. Tais níveis determinam alerta epidemiológico compatível com o conceito de local-sentinela.

Segundo o ponto de vista da dosagem de 2,5 $\mu \mathrm{g} / \mathrm{dL}$ como ponto de corte para gravidade individual de excreção de iodo urinário, três municípios tiveram doses de todas as amostras abaixo deste valor: Paranã (Tocantins), Conceição (Paraíba) e Nova Roma (Goiás). Estes valores podem ser tomados como indicadores de falta aguda de iodo na dieta, com implicações locais imediatas sobre o desenvolvimento das crianças amostradas. Para fins práticos, esta informação é a reconfirmação de dados existentes antes de 1955, sobre a extensão da prevalência de populações sob risco. O estabelecimento de municípios-sentinela em tais áreas seria decorrência natural. Isso não exclui da vigilância os estados do Paraná, Mato Grosso do Sul, Goiás, e Maranhão, onde o problema clínico detectado não se faz acompanhar do critério epidemiológico de risco a partir da dosagem urinária. A distribuição dos dados globais aponta, no entanto, para valores no limite do ingresso na zona de deficiência leve. Do ponto de vista ecológico, a distribuição brasileira é limítrofe. $\mathrm{O}$ valores estaduais medianos abaixo deste limiar foram o Acre, Amapá e Tocantins.

\section{Iodo suplementar no sal residencial}

Foi tentada a mesma apreciação em relação aos níveis de iodação no sal residencial e comercial de consumo humano segundo valores ecológicos por município. A identificação das dosagens por criança não foi viável, mas somente crianças examinadas foram amostradas, o que concretiza a dosagem como estimador confiável do sal residencial das crianças da sub-amostra selecionada. 
TABELA 5. Municípios com prevalência de bócio grave e moderada em escolares de 6 a 14 anos. Inquérito nacional, Brasil, 1994 a 1996

\begin{tabular}{|c|c|c|}
\hline Município & Estado & Total $(\%)$ \\
\hline Barra do Bugres & Mato Grosso & 47,22 \\
\hline Viana & Espírito Santo & 34,69 \\
\hline Jaru & Rondônia & 30,71 \\
\hline Jaraguari & Mato Grosso do Sul & 30,10 \\
\hline Cachoeiro do Itapemirim & Espírito Santo & 27,67 \\
\hline Cristalina & Goiás & 27,11 \\
\hline São José do Rio Claro & Mato Grosso & 27,00 \\
\hline Sambaiba $^{a}$ & Maranhão & 26,23 \\
\hline Corumbá & Mato Grosso do Sul & 25,97 \\
\hline Ouro Preto do Oeste ${ }^{\mathrm{a}}$ & Rondônia & 25,00 \\
\hline Três Lagoas & Mato Grosso do Sul & 24,63 \\
\hline Ji-Paraná & Rondônia & 23,64 \\
\hline Bela Vista & Mato Grosso do Sul & 22,95 \\
\hline Paranaíba & Mato Grosso do Sul & 21,54 \\
\hline Angélica & Mato Grosso do Sul & 21,43 \\
\hline Aquidauana & Mato Grosso do Sul & 21,27 \\
\hline Campo Grande & Mato Grosso do Sul & 21,09 \\
\hline Mirador & Maranhão & 20,37 \\
\hline Paranhos & Mato Grosso do Sul & 20,00 \\
\hline Riachãob & Maranhão & 19,56 \\
\hline Vila Nova do Mamoré & Rondônia & 19,32 \\
\hline \multicolumn{3}{|l|}{ São Raimundo } \\
\hline das Mangabeiras ${ }^{b}$ & Maranhão & 19,07 \\
\hline
\end{tabular}

${ }^{a}$ Municípios vizinhos aos municípios-sentinela.

b Incluídos por serem municípios-sentinela.

FIGURA 1. Prevalência de bócio em escolares de 6 a 14 anos. Inquérito nacional, Brasil, 1994 a 1996

Municípios com prevalência grave.

Municípios com prevalência moderada.

Estados com predomínio de prevalência level.

Estados com municípios de prevalência grave e moderada.
TABELA 6. Número de municípios com prevalência de bócio leve em escolares de 6 a 14 anos. Inquérito nacional, Brasil, 1994

\begin{tabular}{lrr}
\hline \multicolumn{1}{c}{ Estado } & Municípios & \multicolumn{1}{c}{$\%$} \\
\hline Acre & 5 & 6,02 \\
Amazonas & 11 & 13,25 \\
Amapá & 1 & 1,20 \\
Ceará & 4 & 4,81 \\
Espírito Santo & 2 & 2,40 \\
Goiás & 2 & 2,40 \\
Maranhão & 12 & 14,45 \\
Mato Grosso do Sul & 5 & 6,02 \\
Mato Grosso & 9 & 10,84 \\
Pará & 13 & 15,66 \\
Paraíba & 1 & 1,20 \\
Pernambuco & 1 & 1,20 \\
Piauí & 1 & 1,20 \\
Paraná & 11 & 13,25 \\
Rondônia & 3 & 3,61 \\
Sergipe & 1 & 1,20 \\
Tocantins & 1 & 1,20 \\
$\quad$ Total & 83 & 100,00 \\
\hline
\end{tabular}

Foram encontradas dosagens medianas abaixo de $20 \mathrm{mg} / \mathrm{kg}$ de sal em 231 municípios. Destes, 30 tiveram dosagens consideradas gravemente baixas, menores que $10 \mathrm{mg} / \mathrm{kg}$ de sal (conforme portaria 1806 do Ministério da Saúde, de 24 de novembro de 1994, que determina níveis de iodação do sal de consumo humano para prevenção do bócio endêmico). A localização destes municípios - em estados de prevalência grave e moderada de bócio - é particularmente preocupante: Rondônia e Goiás, além de Tocantins, Piauí, Bahia e Rio Grande do Norte, este último um estado produtor onde todo o sal deveria ser iodado. Há, portanto, indícios de distribuição e consumo de sal não iodado em regiões endêmicas, assim como de consumo recente (1994) de sal não iodado no próprio município produtor.

A média de iodo no sal nacional foi de $16,3 \mathrm{mg} / \mathrm{kg}$, com desvio padrão de 8,28 (erro padrão da média $=0,025989$ ). A mediana foi de $15,00(\mathrm{Q} 1=12,5 \mathrm{e}$ $\mathrm{Q} 3=18,8)$. A dosagem máxima encontrada foi de $53,4 \mathrm{mg} / \mathrm{kg}$, quando a faixa legalmente imposta no país à época do inquérito era de 40 a $60 \mathrm{mg} /$ $\mathrm{kg}$ de sal. 
TABELA 7. Bócio e iodo urinário em escolares de 6 a 14 anos. Inquérito nacional, Brasil, 1994 a $1996^{a}$

\begin{tabular}{|c|c|c|c|c|c|c|c|c|c|}
\hline \multirow[b]{3}{*}{ Grau } & \multicolumn{9}{|c|}{ Excreção de iodo urináriob } \\
\hline & \multicolumn{2}{|c|}{ Suficiente } & \multicolumn{2}{|c|}{ Leve } & \multicolumn{2}{|c|}{ Moderada } & \multicolumn{2}{|c|}{ Grave } & \multirow[b]{2}{*}{ Total } \\
\hline & No. & $\%$ & No. & $\%$ & No. & $\%$ & No. & $\%$ & \\
\hline Zero & 4912 & 95,69 & 1479 & 95,36 & 548 & 94,65 & 380 & 97,69 & 7319 \\
\hline Grau I & 211 & 4,11 & 69 & 4,45 & 30 & 5,18 & 9 & 2,31 & 319 \\
\hline Grau II & 10 & 0,19 & 3 & 0,19 & 1 & 0,17 & 0 & 0,00 & 14 \\
\hline Total & \multicolumn{2}{|c|}{5133} & \multicolumn{2}{|c|}{1551} & \multicolumn{2}{|c|}{579} & \multicolumn{2}{|c|}{389} & 7652 \\
\hline
\end{tabular}

a Qui-quadrado $(6 \mathrm{gl})=5,977 ; P=0,426$.

${ }^{b}$ Suficiente $=>10 \mu \mathrm{g} / \mathrm{dL}$; leve $=5-10 \mu \mathrm{g} / \mathrm{dL} ;$ moderada $=2,5-5 \mu \mathrm{g} / \mathrm{dL} ;$ grave $=<2,5 \mu \mathrm{g} / \mathrm{dL}$.

O resultado da apreciação das doses de iodo salino conforme a ocorrência de casos de bócio clínico é mostrado na tabela 9. A análise destes valores a partir dos critérios de risco para estudos transversais, empregando-se a razão de ocorrências (odds ratio, OR) como medida de associação entre bócio e iodação insuficiente do sal de consumo humano, é mostrada na tabela 10. Evidencia-se um risco 1,85 vez maior de uma criança com bócio residir em município com iodação moderada ou gravemente insuficiente do sal de cozinha.

\section{DISCUSSÃO}

Dados coletados a partir de Vitti et al. (4) e Oberhofer et al. (13) e submetidos a meta-análise recente permitiram inferir sensibilidade do exame clínico de palpação e inspeção para diagnóstico de bócio variando de 57 a $76 \%$, com especificidade de 80 a $90 \%$. Considerando prevalências de 3 a $22 \%$, o valor preditivo positivo variou de 40 a $60 \%$ e o valor preditivo negativo de 80 a $90 \%$. Estes valores confirmam as suposições de Gandra (3) sobre a variabilidade de pelo menos $20 \%$ nos estimadores de prevalência com os exames clínicos de inspeção e palpação.

A transição do uso da classificação de Gandra (3), de seis valores (0A, OB, 1, 2, 3, 4), para a classificação de três valores $(0, \mathrm{I}, \mathrm{II})$ permite supor que os examinadores treinados sob os padrões da classificação anterior, ao classificar sob o novo esquema, tenderiam a anotar os bócios visíveis com o pescoço estendido sob o valor I (2). Se

TABELA 8. Dosagens urinárias de excreção de iodo. Inquérito nacional sobre prevalência de bócio em escolares de 6 a 14 anos, Brasil, 1994 a 1996

\begin{tabular}{lrrr}
\hline \multicolumn{1}{c}{ Estado } & Amostras & Média & Mediana \\
\hline Roraima & 96 & 13,34 & 12,4 \\
Acre & 35 & 12,87 & 6,1 \\
Amazonas & 204 & 12,28 & 11,5 \\
Roraima & 30 & 12,20 & 12,2 \\
Pará & 74 & 16,04 & 13,1 \\
Amapá & 160 & 11,74 & 9,8 \\
Tocantins & 754 & 10,98 & 9,4 \\
Maranhão & 396 & 15,44 & 14,3 \\
Piauí & 449 & 14,54 & 13,0 \\
Ceará & 636 & 18,45 & 19,7 \\
Rio Grande do Norte & 404 & 14,81 & 12,6 \\
Paraíba & 329 & 15,15 & 13,3 \\
Pernambuco & 283 & 14,95 & 13,5 \\
Alagoas & 429 & 18,05 & 19,4 \\
Sergipe & 408 & 17,04 & 15,2 \\
Bahia & 196 & 15,25 & 14,5 \\
Minas Gerais & 291 & 15,93 & 15,1 \\
Espírito Santo & 403 & 15,92 & 15,3 \\
Rio de Janeiro & 112 & 15,60 & 14,7 \\
São Paulo & 346 & 16,16 & 15,5 \\
Paraná & 140 & 15,92 & 14,9 \\
Santa Catarina & 429 & 15,42 & 13,7 \\
Rio Grande do Sul & 191 & 19,37 & 19,3 \\
Mato Grosso do Sul & 423 & 16,24 & 14,3 \\
Goiás & 434 & 14,67 & 13,6 \\
Total & 7652 & 15,34 & 14,0 \\
\hline
\end{tabular}

esta suposição for válida, o grau II poderia estar subestimado, e o grau I, superestimado, dependendo do número de entrevistadores treinados sob o esquema abandonado. Deve-se notar que ambas as classificações estavam presentes na folha de anotações levada a campo no inquérito, o que reforça estas hipóteses. A discussão sobre regras classificatórias e seu impacto em inquéritos pode ser encontrada igualmente no campo internacional (14).
A tomada de medidas palpativas e visuais do tamanho linear da glândula tireóide baseou-se, entre outros referenciais, em sistema que pressupõe o aumento em tamanho linear para o dobro ou até quatro a cinco vezes o tamanho usual desta glândula. Problemas de validade e precisão destes padrões sempre foram motivo de apaixonadas discussões entre especialistas da área. A raiz das discussões está em que aumentos lineares para duas vezes 
TABELA 9. Valores nacionais de dosagens de iodo salino e bócio clínico em crianças de 6 a 14 anos. Inquérito nacional, Brasil, 1994 a $1996^{\mathrm{a}}$

\begin{tabular}{|c|c|c|c|c|c|c|c|c|c|}
\hline \multirow[b]{3}{*}{ Grau } & \multicolumn{9}{|c|}{ lodação salinab } \\
\hline & \multicolumn{2}{|c|}{ Suficiente } & \multicolumn{2}{|c|}{ Leve } & \multicolumn{2}{|c|}{ Moderada } & \multicolumn{2}{|c|}{ Grave } & \multirow[b]{2}{*}{ Total } \\
\hline & No. & $\%$ & No. & $\%$ & No. & $\%$ & No. & $\%$ & \\
\hline Zero (normal) & 1967 & 97,52 & 18293 & 97,38 & 61497 & 95,14 & 15450 & 95,85 & 97207 \\
\hline | e || & 50 & 2,48 & 493 & 2,62 & 3140 & 4,86 & 669 & 4,15 & 669 \\
\hline Total & \multicolumn{2}{|c|}{2017} & \multicolumn{2}{|c|}{18786} & \multicolumn{2}{|c|}{64637} & \multicolumn{2}{|c|}{16119} & 101559 \\
\hline
\end{tabular}

a Qui-quadrado $(3 \mathrm{gl})=194,7 ; P>0,0001$.

${ }^{\mathrm{b}}$ Suficiente = > $40 \mathrm{mg} / \mathrm{kg}$; leve $=20-40 \mathrm{mg} / \mathrm{kg}$; moderada $=10-20 \mathrm{mg} / \mathrm{kg} ;$ grave $=<10 \mathrm{mg} / \mathrm{kg}$.

\begin{abstract}
TABELA 10. Valores nacionais de dosagens de iodo salino em faixas de adequação e bócio clínico em crianças de 6 a 14 anos. Inquérito nacional, Brasil, 1994 a $1996^{a}$
\end{abstract}

\begin{tabular}{lccr}
\hline & \multicolumn{3}{c}{ Presença de bócio em crianças } \\
\cline { 2 - 4 } lodação salina & Bócio & Normal & \multicolumn{1}{c}{ Total } \\
\hline Moderada e grave & 3809 & 76947 & 80756 \\
Leve e suficiente & 543 & 20260 & 20803 \\
$\quad$ Total & 4352 & 97207 & 101559 \\
\hline a Qui-quadrado (não corrigido) $(1 \mathrm{gl})=$ & \multicolumn{1}{c}{178,$96 ; P>0,0000001$. OR $=1,85 ; 1,68<$ OR $<2,03$} \\
IC 95\% Cornfield.
\end{tabular}

o tamanho normal implicam em aumento volumétrico de oito a 10 vezes em relação ao volume normal de referência. Tomando-se como exemplo os lóbulos da tireóide de adultos, medidas de $4,0 \times 2,5 \times 0,5 \mathrm{~cm}(5 \mathrm{~mL})$, ao passar para $8,0 \times 5,0 \times 1,0 \mathrm{~cm}$, implicam em volume aproximado de $40 \mathrm{~mL}$. Em inquéritos conduzidos com recursos ecográficos, o volume normal da tireóide em crianças de 8 a 14 anos pode variar de 3 a $6,5 \mathrm{~mL}$ (13), sendo que os limites do percentil 97 desta distribuição são colocados em 4,5 e 10,5 mL, respectivamente $(15,16)$. A tendência da década de 1990 foi enfatizar métodos de imagem por ultra-som, que fornecem estimativas volumétricas diretas, além dos contornos exatos da massa ecogênica específica. Contudo, a utilização de aparelhos do tipo sonar-doppler em inquéritos de massa é dificultada em função do alto custo associado (6).

No inquérito nacional brasileiro de 1994, optou-se por utilizar o método de melhor custo, mas de piores condições de validação. Esta opção foi motivo de acaloradas discussões so- bre as conseqüentes necessidades de controle de qualidade, através de técnicas conhecidas pela epidemiologia, a saber: padronização exaustiva em cascata dos multiplicadores de treinamento, com re-exame por árbitros participantes do treinamento no primeiro nível (central); recaptura de amostras aleatórias para reavaliação durante e após a coleta de dados no campo; captura de populações vizinhas não amostradas para exame do grau de concordância regional, local e interequipes; recaptura de populações com valores aberrantes (por exemplo, Cristalina, Estado de Goiás); estudo no campo durante a coleta comparando diariamente as médias de detecção por equipe; troca de equipes entre áreas díspares para avaliar gradientes locais de deteç̧ão; e outras formas rotativas ou de recaptura que visam o controle de qualidade durante e imediatamente após a coleta. Esses são fatores limitantes da análise dos presentes resultados, pois tais métodos não foram aplicados no inquérito.

Os resultados mostram que o polígono geográfico do bócio endêmico conhecido nos anos 1980 e 1990 (17) foi estendido para o extremo norte do Brasil. A detecção de prevalências leves, moderadas e graves de bócio endêmico em escolares de 6 a 14 anos se expandiu, no período de 1990 a 1994, para as regiões do oeste da Amazônia, incluindo os estados do Amazonas, Acre e Rondônia. Foram outra vez detectadas, após antigos registros de 1955 (18), prevalências graves na região de colonização e expansão agrícola do eixo da rodovia BR-364 (Cuiabá-Porto Velho).

A superposição das distribuições de excreção urinária em regiões endêmicas e não endêmicas não autoriza a aplicar a cada criança individualmente o perfil de doses encontrado para o grupo, havendo reservas, do ponto de vista clínico, quanto à interpretação do dado isolado. É, no entanto, um indicador precoce do perfil populacional e pode ser utilizado para dimensionar a população sob risco de bócio. Sua utilidade aparenta ser estritamente epidemiológica, uma vez que há crianças na amostra com dosagens baixas mesmo em estados considerados de bons índices de excreção. O panorama descrito é sobretudo ecológico, tendo a ver com a tentativa de estimar populações sob risco de bócio, dependendo da validade das dosagens urinárias, da dieta natural e da efetividade e eficácia da suplementação de iodo no sal de cozinha comercializado em território brasileiro.

Deve-se ressaltar que esta apreciação está ambientada na realidade da suplementação de iodato de potássio em sal de cozinha no Brasil em nível apontado como nacionalmente 
efetivo e permanente, embora em quantidade (concentração) insuficiente. Os dados deste inquérito foram coletados em 1994, período no qual não há notícia pública sobre interrupção da iodação nas indústrias que preparam o sal para distribuição em todo o território nacional.

\section{CONCLUSÕES}

Houve queda de $86 \%$ na prevalência do bócio em relação à prevalência mediana detectada em 1975 - de 12\% para $1 \%$ em todos os estados brasileiros. São Paulo, Minas Gerais e Bahia deixaram de ser endêmicos. O bócio é problema de saúde não localizado ou sem região endêmica definida, com municípios de maior prevalência nas regiões Centro-Oeste e Norte e respectivas fronteiras com a região Nordeste.

Durante o período estudado, $12 \%$ das crianças apresentaram dosagens de iodo urinário abaixo de $5 \mu \mathrm{g} / \mathrm{dL}$, implicando a criação de ambiente de alto risco para ocorrência de bócio em crianças, adolescentes e nascituros nos anos seguintes sob ausência de intervenção especial. Os dados de excreção urinária não revelam estado carencial que diferencie as crianças portadoras de bócio das normais, embora seja lícito supor que todas estivessem submetidas ao mesmo tipo de dieta e condições ambientais, havendo bócio naquelas em quem a suscetibilidade individual determinou maior fragilidade.

Como o risco de apresentar bócio em crianças de 6 a 14 anos foi duas vezes maior nas áreas onde a iodação do sal foi deficiente $(<10 \mathrm{mg} / \mathrm{kg})$, devem-se associar ações de suplementação de iodato de potássio no sal de consumo humano com as de vigilância à saúde em programas locais. Há indicações de expansão das áreas endêmicas conhecidas para as regiões de abertura de fronteira agrícola recente, sendo que crianças estavam sendo submetidas a dieta doméstica carente de iodo veiculado pelo sal. Esta carência de suplemento, política e economicamente determinada, é verificável pela tendência de distribuição de maior número de casos de bócio entre crianças submetidas a dietas carenciais.

Recomenda-se que os municípios com prevalência de $20 \%$ ou mais de bócio sejam incluídos intencionalmente em novo inquérito. Nas áreas de alta prevalência de bócio deve ser feito rastreamento das rotas de distribuição de sal visando o controle do bócio endêmico. Inquéritos posteriores devem adotar técnicas de medidas de volume tireiodiano por ecografia ultra-sônica associada a medidas de iodo urinário.

Agradecimentos. A equipe agradece o apoio na coleta de dados por parte dos pesquisadores, técnicos, monitores e pessoal estratégico do Instituto Nacional de Alimentação e Nutrição/Mato Grosso do Sul; da Fundação Nacional de Saúde/Mato Grosso do Sul; e, em particular, agradecemos ao Professor Fernando Barros (Universidade Federal de Pelotas/Rio Grande do Sul); Professor Yaro Ribeiro Gandra (Universidade de São Paulo); Professora Denise Costa Coitinho (Universidade de Brasília e Ministério da Saúde); Professor Rui Maciel (Universidade Federal de São Paulo); Dra. Denise Franco (Secretaria de Saúde do Distrito Federal); e Drs. Jean Pedreira, José de Ribamar Bulcão, Sebastião Gonçalves de Lima e Elizabeth Silva Araújo (Fundação Nacional de Saúde/ Mato Grosso do Sul).

\section{REFERÊNCIAS}

1. Medeiros-Filho A. Bócio endêmico: levantamento de sua prevalência em todo o território brasileiro por micro-regiões homogêneas. Rev Bras Malariol D Trop 1976;28(1/4):1-49.

2. World Health Organization/UNICEF/International Council for the Control of Iodine Deficiency Disorders. Global prevalence of iodine deficiency disorders. Geneva: WHO; 1993. (MDIS Working Paper 1).

3. Gandra YR. Avaliação clínica do bócio endêmico. Rev Saude Publ 1984;18:396-404.

4. Vitti P, Martino E, Aghini-Lombardi F, Rago T, Antonangeli L, Maccherini D, et al. Thyroid volume measurement by ultrasound in children as a tool for the assessment of mild iodine deficiency. J Clin Endocrinol Metab 1994; 79(2):600-603.

5. Brasil, Ministério da Saúde, Fundação Nacional de Saúde, Instituto Nacional de Alimentação e Nutrição. Manual de combate aos distúrbios por deficiência de iodo no Brasil. Brasília: Fundação Nacional de Saúde; 1996.
6. World Health Organization. Indicators for assessing iodine deficiency disorders and their control programmes. Geneva: WHO; 1993. (Nutrition Committee Working Paper 93.1).

7. Hetzel BS, ed. United Nations Administrative Committee on Coordination, Subcommittee on Nutrition. The Prevention and control of iodine deficiency disorders. ACC/SCN Stateof-the-Art series. Rome: ACC/SCN; 1988. (Nutrition Policy Discussion Paper 3).

8. Dunn JT, Crutchfield HE, Gutenkunst R, Dunn AD. Two simple methods for measuring iodine in urine. Thyroid 1993;3(2):119123.

9. Tukey JW. Exploratory data analysis. Reading, Massachusetts: Addison-Wesley Publishing Co; 1977.

10. Brasil, Ministério da Saúde, Instituto Nacional de Alimentação e Nutrição, Superintendência de Campanhas de Saúde Pública. Programa de combate ao bócio endêmico. Brasília: Instituto Nacional de Alimentação e Nutrição; 1969.
11. Brasil, Ministério da Saúde, Instituto Nacional de Alimentação e Nutrição, Superintendência de Campanhas de Saúde Pública. Programa de combate ao bócio endêmico. Brasília: Instituto Nacional de Alimentação e Nutrição; 1989.

12. Esteves RZ. Determinação da excreção urinária de iodo em escolares brasileiros [tese de doutorado]. São Paulo: Universidade Federal de São Paulo/Escola Paulista de Medicina: 1997.

13. Oberhofer R, Ober A, Seeber A, Amor H. Struma-Epidemiologie in Südtirol. Dtsch Med Wschr Stuttgart 1992;117(40):1508-1512.

14. Delange F, Bastani S, Benmiloud M, DeMaeyer E, Isayama MG, Koutras D, et al. Definitions of endemic goiter and cretinism, classification of goiter size and severity of endemics, and survey techniques. Em: Dunn JT, Pretell EA, Daza CH, Viteri FE, eds. Towards the eradication of endemic goiter, cretinism, and iodine deficiency. Washington, D.C.: Pan 
American Health Organization/WHO; 1986. (Scientific Publication 502). Pp. 373-376.

15. World Health Organization/International Council for Control of Iodine Deficiency Disorders. Update: recommended normative values for thyroid volume in children aged 6-15 years. Bull World Health Org 1997;75(2):9597.

16. Querido A, Delange F, Dunn JT, FierroBenitez R, Ibbertson HK, Kourtas DA, et al. Definitions of endemic goiter and cretinism: Classification of goiter size and severity of en- demics and survey techniques. Em: Dunn JT, Medeiros-Neto GA, eds. Endemic goiter and cretinism: continuing threats to world health. Washington, D.C.: Pan American Health Organization; 1974. Pp. 267-272. (Scientific Publication 292)

17. Dantas LM. Endemic goiter in Brazil. Em: Dunn JT, Pretell EA, Daza CH, Viteri FE, eds. Towards the eradication of endemic goiter, cretinism, and iodine deficiency. Washington, D.C.: Pan American Health Organization/WHO; 1986. Pp. 271-275. (Scientific Publication 502).
18. Medeiros-Neto GA. Cento e oitenta anos de bócio endêmico no Brasil. Arq Bras Endocrinol Metabol 1982;26(4):120-128.

Manuscrito recebido em 10 de dezembro de 2001. Aceito em versão revisada em 5 de agosto de 2002.

ABSTRACT Objective. To determine the national and state-level prevalence of endemic goiter associated with iodine deficiency in schoolchildren between 6 and 14 years of age in Brazil, based on data from a national survey carried out from 1994 to 1996.

Endemic goiter prevalence survey in Brazilian schoolchildren 6 to 14 years old, 1994-1996
Materials. Thyroid inspection and palpation were performed on 178774 schoolchildren between 6 and 14 years of age, in all the states of Brazil. A subsample was selected to measure the iodine content in their urine as well as the iodine content of the table salt used for cooking in their homes.

Results. The last survey before this one was carried out in 1975 . We found an $86 \%$ decrease in the median prevalence of grade 1 and grade 2 goiter, from $12 \%$ to $1 \%$. In $21 \%$ of the municipalities, the observed prevalence was zero. From the 16803 urine samples collected for iodine measurement, 7702 were matched with the corresponding clinical record (a loss of 54\%). The median urinary iodine level was $14.0 \mu \mathrm{g} / \mathrm{dL}$, and without a significant correlation with the clinical data on goiter. The median iodine excretion level for the population in the states of Acre, Amapá, and Tocantins was equal to or below $9.0 \mu \mathrm{g} / \mathrm{dL}$. In three municipalities, urinary iodine was below $2.5 \mu \mathrm{g} / \mathrm{dL}$ in all the samples: Paranã (in the state of Tocantins), Conceição (Paraíba), and Nova Roma (Goiás). The supplementary iodine found in the salt collected from households (458 samples collected) was below recommended levels, with $50 \%$ of them below $20 \mathrm{mg} / \mathrm{kg}$ (20 ppm). In 7\% of the samples, the level of iodine was below $10 \mathrm{mg} / \mathrm{kg}$ of salt, even in salt-producing states such as Rio Grande do Norte.

Conclusions. There is evidence that endemic goiter is expanding to parts of Brazil that more recently have been opened up for agriculture, with children in those areas being exposed to low levels of supplementary iodine in the salt used for cooking. We found an increased risk of goiter in areas with deficient iodine supplementation $(<10 \mathrm{mg} / \mathrm{kg})$ (odds ratio $=1.85$; Cornfield 95\% confidence interval: 1.68-2.03). 